\title{
Collaborative Decision-Making Challenges in the Dutch Railway System
}

\author{
N. Jakubeit ${ }^{(\bowtie)}$, M. Rajabalinejad, A. J. J. Braaksma, and L. A. M. van Dongen \\ University of Twente, De Horst 2, 7522LW Enschede, The Netherlands \\ n.jakubeitautwente.nl
}

\begin{abstract}
In the context of systems integration, the railway system needs to pay more attention to organizational interoperability. Companies along the value chain collaborate at the interfaces to accomplish the shared purposes of the system. These collaborations between different stakeholders having different interests and backgrounds demonstrate a demand for an integral approach towards decision-making. This paper investigates in which environment of system change projects within the Dutch Railway system, shared awareness between decision-makers could possibly enhance collaborative decisions. First, a literature review on organizational interoperability and system performance boundaries was conducted. Moreover, the concept of shared situational awareness was introduced to identify its applicability in an organizational context. Afterwards, interviews with key stakeholders of the Dutch Railway system were carried out to recognize the environment in which shared awareness may facilitate integral decision-making. It shows application possibilities in an organizational setting, keeping in mind certain constraints imposed by the environment.
\end{abstract}

